\title{
Phase I clinical trial of vaccination with LY6K-derived peptide in patients with advanced gastric cancer
}

\author{
Hajime Ishikawa $\cdot$ Motohiro Imano $\cdot$ Osamu Shiraishi • \\ Atsushi Yasuda • Ying-Feng Peng • Masayuki Shinkai • \\ Takushi Yasuda $\cdot$ Haruhiko Imamoto $\cdot$ Hitoshi Shiozaki
}

Received: 28 August 2012/Accepted: 24 March 2013/Published online: 24 April 2013

(C) The International Gastric Cancer Association and The Japanese Gastric Cancer Association 2013

\begin{abstract}
Background Lymphocyte antigen 6 complex locus $\mathrm{K}$ (LY6K) has been identified as a tumor-associated antigen in lung cancers and esophageal squamous cell carcinomas. The immunogenicity of LY6K-177 peptide vaccine therapy has been demonstrated in patients with advanced esophageal cancer. This study extends this treatment to gastric cancer.

Methods LY6K expression in clinical samples obtained from gastric cancer patients was examined by immunochemistry. As a phase I clinical trial, the safety and immunogenicity of LY6K-177 peptide vaccine emulsified with Montanide ISA 51 was evaluated in six patients with unresectable advanced gastric cancer. LY6K-177 peptide ( $1 \mathrm{mg}$ in $1 \mathrm{ml}$ sterile saline) was emulsified with incomplete Freund's adjuvant $(1 \mathrm{ml})$ and intracutaneously administered to the inguinal region or axilla. One treatment course comprised four vaccinations, performed weekly for the first and second treatment courses and biweekly for the third treatment course.

Results LY6K expression was confirmed in $85 \%$ of gastric cancer tissues. Induration and redness at the vaccination site (grade I), possibly a delayed-type hypersensitivity reaction, was observed in all patients; however, no systemic toxicology was identified in any patient throughout the observation period. Three of the six patients had stable disease, and a tumor contraction effect was observed in one patient.
\end{abstract}

H. Ishikawa ( $\square) \cdot$ M. Imano · O. Shiraishi · A. Yasuda · Y.-F. Peng · M. Shinkai - T. Yasuda - H. Imamoto ·

H. Shiozaki

Department of Surgery, Kinki University, 377-2 Ohno-higasi, Osaka-sayama, Osaka 589-8511, Japan

e-mail: haj.02@hotmail.co.jp; hajime@surg.med.kindai.ac.jp
Conclusions LY6K was expressed in $85 \%$ of observed gastric cancers. Vaccination with LY6K-177 peptide/ Montanide ISA 51 appeared to be tolerated by advanced gastric cancer patients, and moreover anticancer efficacy was suggested. This trial was registered with ClinicalTrial.gov (no. NCT00845611).

Keywords LY6K · Gastric cancer · Peptide vaccine · Immunotherapy

\section{Introduction}

Gastric cancer is the second leading cause of death from malignant disease worldwide [1], although the incidence and mortality rate have decreased significantly in recent years [2]. The cumulative 5-year survival rate is $68.2 \%$ (89.9\% for stage I, $69.1 \%$ for stage II, $43.5 \%$ for stage III, $9.9 \%$ for stage IV patients) [3]. Although resection is the most common therapy, surgery alone is insufficient for advanced gastric cancer. Use of the chemotherapeutic drug S-1 together with other agents has improved treatment results for advanced gastric cancer, with the mean survival time exceeding 1 year, although it remains in a range of 12.6-14.4 months [4-6]. New therapies for the treatment of advanced gastric cancer are therefore required.

Systematic cDNA microarray analysis of the expression levels of thousands of genes in lung cancer and esophageal squamous cell carcinoma (ESCC) tissues compared with normal human tissues [7-11] revealed that lymphocyte antigen 6 complex locus K (LY6K) expresses a tumorassociated antigen (TAA). LY6K is a typical cancer/testis antigen, highly expressed in $88.2 \%$ of surgically resected non-small cell lung carcinomas (NSCLC) and $95.1 \%$ of ESCCs [12]. Suppression of LY6K expression with siRNA 
effectively inhibits the growth of LY6K-expressing lung and esophageal cancer cells, indicating that it might be associated with a highly malignant phenotype [12]. Furthermore, human leukocyte antigen (HLA)-A*2402-positive dendritic cells pulsed with a LY6K-derived epitope peptide, LY6K peptide (RYCNLEGPPI), was shown to induce $\mathrm{CD} 8+$ cytotoxic $\mathrm{T}$ lymphocytes (CTL) to exert specific cytotoxicity against HLA-A*2402-positive LY6Kexpressing lung and esophageal carcinoma cell lines [12]. Thus, LY6K-177 could be useful for inducing specific immune responses in the treatment of NSCLC and ESCC.

The immunogenicity of LY6K-177 peptide vaccine therapy in patients with unresectable advanced esophageal cancer has also been demonstrated [13, 14]. We obtained similar results in an esophageal cancer patient, raising hopes for a new treatment option using this peptide. If LY6K is also expressed at a high level in gastric cancers, a vaccine therapy similar to that for esophageal cancer might be possible. Since confirming LY6K expression in gastric cancer, we have also treated stomach cancer patients using an HLA-A*2402restricted LY6K-derived epitope peptide vaccine.

\section{Materials and methods}

Analysis of LY6K expression in gastric cancer

Clinical samples were obtained from gastric cancer patients who underwent surgery from 2001 to 2007 and were subsequently diagnosed as stage IIIa or IIIb based on the Japanese Classification of Gastric Carcinoma (13th edition, June 1999). To determine the status of LY6K protein in clinical gastric cancer samples embedded in paraffin blocks, we performed immunohistochemistry as described previously $[12,15,16]$. Briefly, rabbit polyclonal antihuman LY6K antibodies (TM38) were applied to the sections after blocking endogenous peroxidases and proteins. The samples were then incubated with HRP-labeled anti-rabbit IgG (secondary antibody), and substrate chromogen was added. The specimens were counterstained with hematoxylin. LY6K positivity was assessed semiquantitatively without prior knowledge of clinicopathological data. The intensity of LY6K staining was evaluated using the following criteria: dark brown staining that completely obscured the cytoplasm in $>50 \%$ of tumor cells, strong positive $(2+)$; any lesser degree of brown staining, weak positive $(1+)$; and no appreciable staining, absent (0). Clinical samples of patients included in the trial also were evaluated for LY6K expression in the same way.

Study design

This phase I clinical study was approved by the Kinki University Institutional Review Board and is open to the public at ClinicalTrial.gov (NCT00845611). The primary endpoint was safety, and the secondary endpoints were therapeutic efficacy and estimation of immunological responses. The LY6K-177 peptide (1 mg in $1 \mathrm{ml}$ sterile saline) was emulsified with $1 \mathrm{ml}$ incomplete Freund's adjuvant (Montanide ISA 51 VG; Seppic, France), and then intracutaneously administered to the inguen or axilla. One treatment course consisted of four vaccinations, performed weekly for the first and second treatment courses and biweekly for the third. Patients who completed at least one treatment course were included in the evaluation of safety and efficacy. A total of six patients were included.

Patient selection

Inclusion criteria:

1. Patients with advanced or recurrent gastric cancer for whom treatment had failed, who were in a situation where effective therapy was not available, or for whom treatment was not possible because of severe adverse effects of chemotherapy

2. A World Health Organization performance status of 0-2

3. Age $\geq 20$ years and $\leq 80$ years

4. Patients with disease for which a treatment effect was judged possible, even if the disease was not measurable

5. More than 2 weeks since previous treatment

6. Expected survival of at least 3 months

7. $\mathrm{WBC} \geq 1,500 / \mathrm{mm}^{3} ; \quad \mathrm{WBC} \leq 15,000 / \mathrm{mm}^{3}$; platelet count $\geq 50,000 / \mathrm{mm}^{3}$; total bilirubin $\leq 3 \times$ the institutional normal upper limit; AST, ALT $\leq 3 \times$ the institutional normal upper limit; creatinine $\leq 3 \times$ the institutional normal upper limit

8. HLA-A*2402 positive

9. Able and willing to give valid written informed consent.

Exclusion criteria:

1. Pregnancy, possible pregnancy, and breastfeeding

2. Serious infection requiring antibiotics

3. Concurrent treatment with steroids or immunosuppressive agents

4. Deemed unsuitable by the principal investigator or physician in charge.

Characteristics of the six patients are shown in Table 1.

Safety assessment

Adverse events were assessed after each treatment course for the duration of vaccine treatment and graded according to NCI CTCAE version 3. 


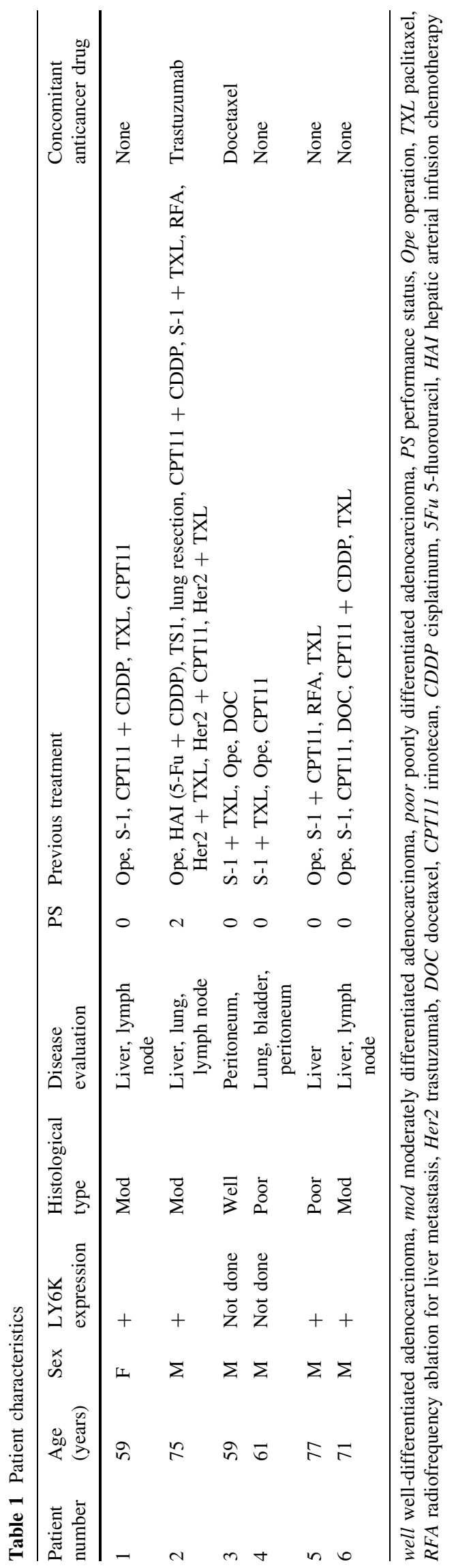

IFN- $\gamma$ ELISPOT assay

Before treatment and at the end of each treatment course, peripheral blood mononuclear cells (PBMCs) were obtained and immediately frozen. For immune monitoring, all frozen PBMCs from each patient were thawed at the same time. Cells $\left(5 \times 10^{5} / \mathrm{ml}\right)$ were stimulated with $10 \mu \mathrm{g} / \mathrm{ml} \mathrm{LY} 6 \mathrm{~K}-177$ peptide and $20 \mathrm{IU} / \mathrm{ml}$ of interleukin2 (Chiron, Emeryville, CA, USA) at $37{ }^{\circ} \mathrm{C}$ under $5 \%$ $\mathrm{CO}_{2}$ for 2 weeks. After depletion of $\mathrm{CD} 4+$ cells, as determined using a Dynal CD4 Positive Isolation Kit (Invitrogen, Carlsbad, CA, USA), cells were assayed by interferon-gamma (IFN- $\gamma$ ) enzyme-linked immunospot (ELISPOT) according to the manufacturer's instructions (BD Biosciences, San Jose, CA, USA). Briefly, HLAA*2402-positive B-lymphoblast TISI cells (IHWG Cell and Gene Bank, Seattle, WA, USA) were incubated overnight with $20 \mu \mathrm{g} / \mathrm{ml}$ LY6K-177 peptide or HIV-Env peptide. Peptides that did not bind to HLA-A*2402 on TISI cells were washed away before the ELISPOT assay. Respective peptide-pulsed TISI cells $\left(2 \times 10^{4}\right.$ cells/well $)$ were used to stimulate prepared CD4-cells $\left(1 \times 10^{4}\right.$ cells/well) in 96-well plates (Millipore, Bedford, MA, USA) at $37{ }^{\circ} \mathrm{C}$ under $5 \% \mathrm{CO}_{2}$ overnight. The plates were then analyzed using an ImmunoSpot S4 Analyzer and ImmunoSpot image analyzer software version 5.0 (Cellular Technology, Cleveland, OH, USA). The number of LY6K peptide-specific spots was calculated by subtracting the number of spots observed after HIV-Env peptide stimulation from that after LY6K-177 peptide stimulation. All ELISPOT assays were performed using triplicate wells.

\section{Clinical monitoring}

Antitumor effects were evaluated after each treatment course based on the RECIST guidelines in patients in whom one vaccine course was completed.

\section{Results}

LY6K expression in gastric cancers

Primary tumor samples from 60 gastric cancer patients were evaluated for LY6K expression by immunohistochemistry. LY6K expression was confirmed in $85 \%$ of the samples, with only 9 showing no expression. In the LY6Kpositive cancer tissues, positive and negative cells coexisted at various ratios (Fig. 1). Associations between the intensity of LY6K staining and the clinical data were subsequently evaluated. The intensity of LY6K staining had no apparent correlation with patient sex, age, 
Fig. 1 Gastric cancer tissue samples from 60 gastric cancer patients were evaluated by immunohistochemistry for LY6K expression. LY6K expression was confirmed in $85 \%$ of the samples

immunohistochemical evaluation of LY6K protein in gastric cancer tissues.

Weak positive

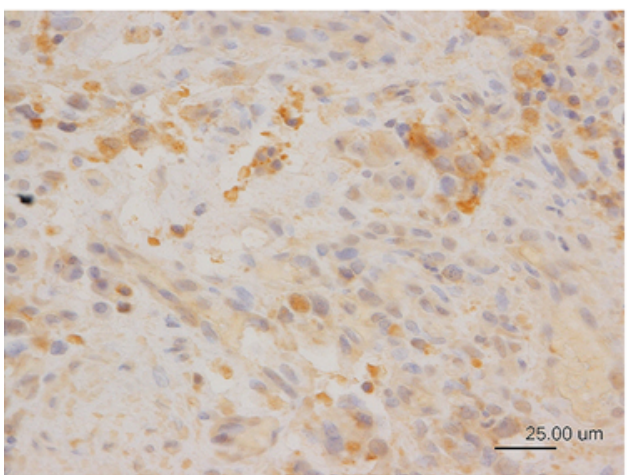

Strong positive

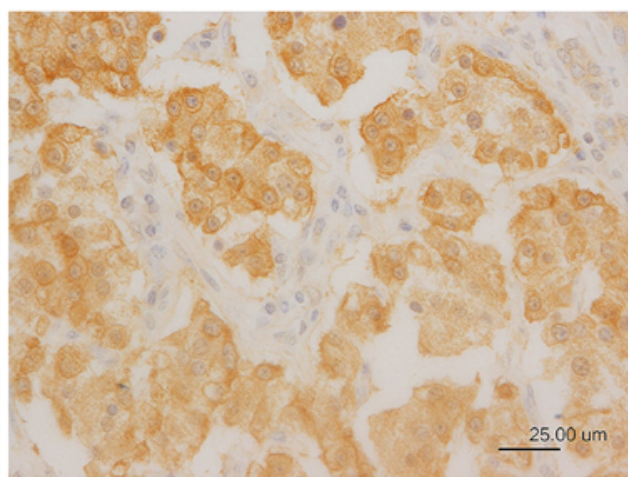

histological type of gastric cancer, or prognosis. However, the fact that $85 \%$ of the gastric cancer tissue was positive for LY6K was taken as a justification for initiating the clinical trial.

\section{Patient characteristics}

All six patients included in the trial had unresectable gastric cancer, had undergone surgery for excision of the primary lesion, and had previously received chemotherapy (Table 1). Lesions were detected by computed tomography in all patients except patient 3, in whom peritoneal dissemination was found during surgery, suggesting that the tumor marker arose because of recurrence of peritoneal dissemination. After ending treatment, the patient died as a result of the peritoneal dissemination.

The tissue samples of gastric cancer obtained from patients 1, 2, 5, and 6 had LY6K expression. In patients 3 and 4 , the amount of tissue required for immunohistochemistry was not obtained for neoadjuvant chemotherapy.

One of the inclusion criteria in the present study was no response to chemotherapy. In standard practice, chemotherapy is discontinued or the agent is changed when chemotherapy proves ineffective. Therefore, it would have been both unconventional and meaningless to continue chemotherapy in the trial. However, two patients hoped to continue chemotherapy despite its failure, so we combined the treatment with vaccine instead of denying the patients' requests. Trastuzumab was administered concurrently to patient 2 , and docetaxel was administered concurrently to patient 3 (Table 1).

\section{Toxicity}

Patients were vaccinated with LY6K-177 peptide/Montanide ISA 51 6-12 times, with an average vaccination frequency of 9.5 (Table 2). Induration and redness at the vaccination site (grade I), possibly a delayed-type hypersensitivity (DTH) reaction, were observed in all patients
Table 2 Summary of vaccine effects

\begin{tabular}{llllll}
\hline $\begin{array}{l}\text { Patient } \\
\text { number }\end{array}$ & $\begin{array}{l}\text { Antitumor } \\
\text { effect }\end{array}$ & $\begin{array}{l}\text { Frequency of } \\
\text { administration }\end{array}$ & DTH & $\begin{array}{l}\text { ELISPOT } \\
\text { assay } \\
\text { (R/S ratio } \\
1)\end{array}$ & $\begin{array}{l}\text { Overall } \\
\text { survival } \\
\text { (days) }\end{array}$ \\
\hline 1 & SD & 12 & + & 129.3 & 263 \\
3 & SD & 8 & + & 135.0 & 302 \\
4 & SD & 12 & + & 129.3 & 362 \\
5 & None & 12 & + & 687.0 & 260 \\
2 & None & 6 & + & 26.6 & 157 \\
6 & None & 7 & + & 17.6 & 78 \\
\hline
\end{tabular}

DTH delayed-type hypersensitivity

(Table 2); however, no systemic toxicology was identified in any patient throughout the observation period. Thus, vaccination with LY6K-177 peptide/Montanide ISA 51 appeared to be tolerated by the advanced gastric cancer patients.

\section{IFN- $\gamma$ ELISPOT assay}

LY6K-177-specific CTL responses were determined by IFN- $\gamma$ ELISPOT assay. The grade of immunity was indicated by specific spots, the average number of spots after stimulation with HIV-Env peptide minus that after stimulation with the LY6K-177 peptide; the R/S ratio of each patient. In each patient, the timing of appearance of the largest spots was adopted, and in all patients, specific spots increased after vaccination. The most remarkable reactions were observed in patients 1, 3, 4, and 5 (Fig. 2). Reliability of this assay was thought to be high because specific spots were clearly correlated with the R/S ratio in all patients.

\section{Clinical outcomes and immunological responses}

The median overall survival (MOS) in this study was 7.9 months. In three of the six patients, the best overall 

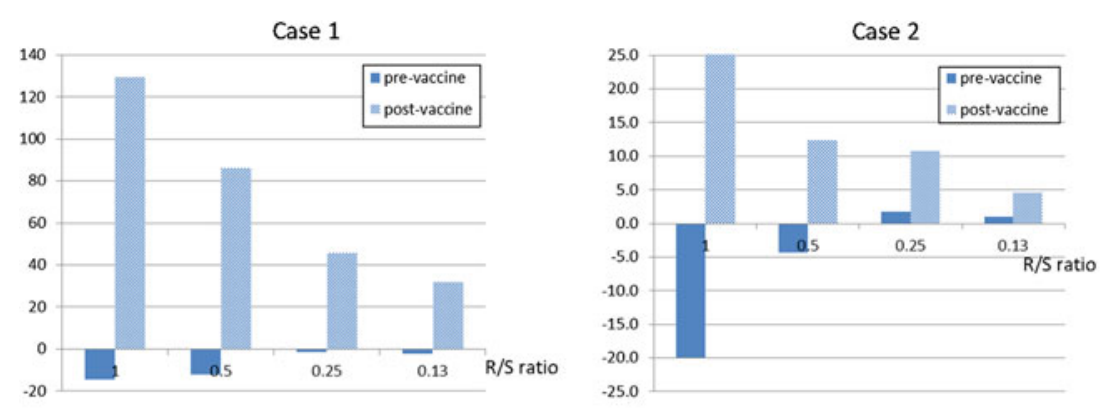

Case 3
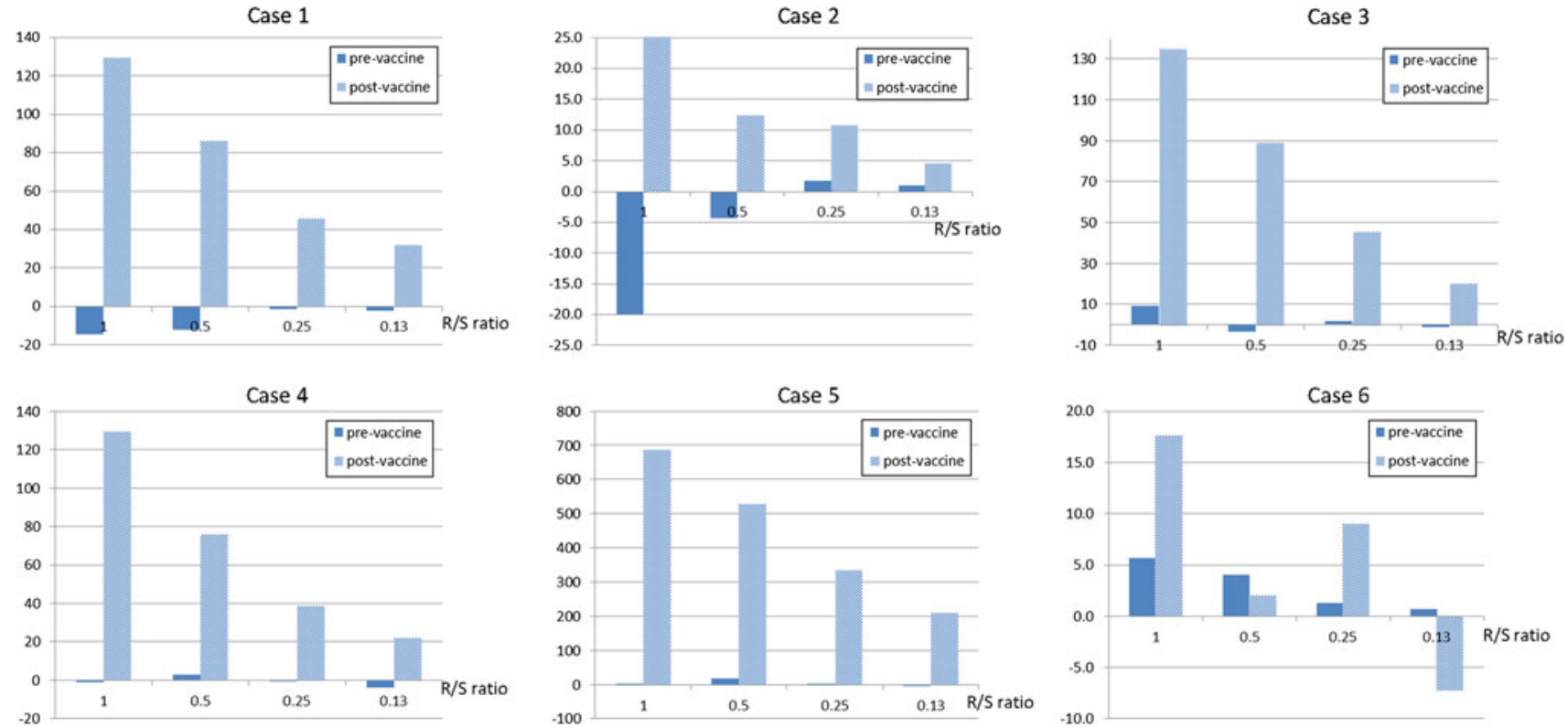

Fig. 2 Number of LY6K peptide-specific spots in IFN- $\gamma$ ELISPOT assay. A significantly higher number of spots was observed in patients 1, 3, 4, and 5 . Reliability of this assay was thought to be high because specific spots were clearly correlated with the R/S ratio in all patients

Fig. 3 Computed tomography showed that evaluated tumors (1-4) were diminished and tumor (5) was enlarged after the first treatment course

\section{CT of case 1}

Before treatment
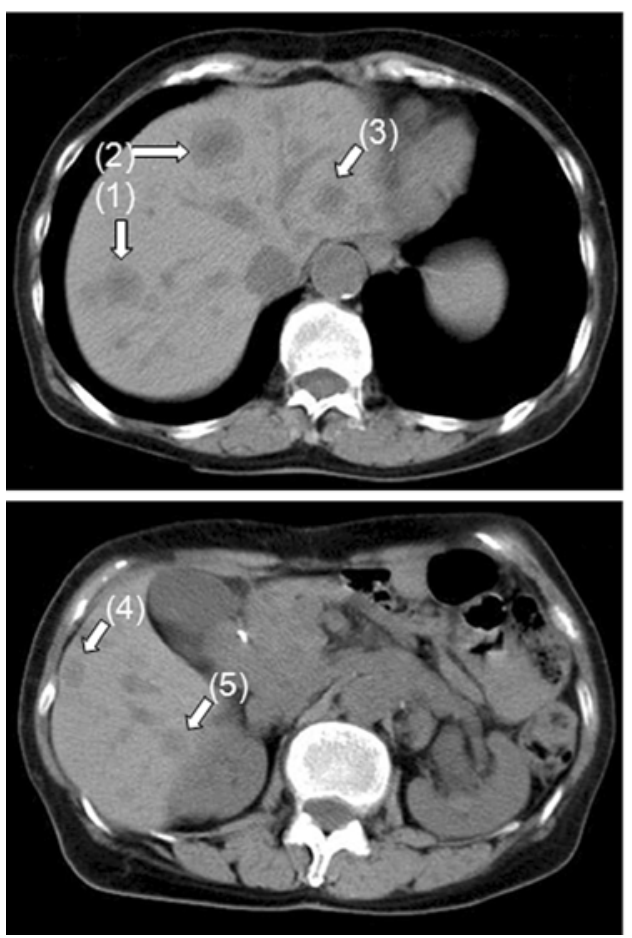

After $1^{\text {st }}$ course
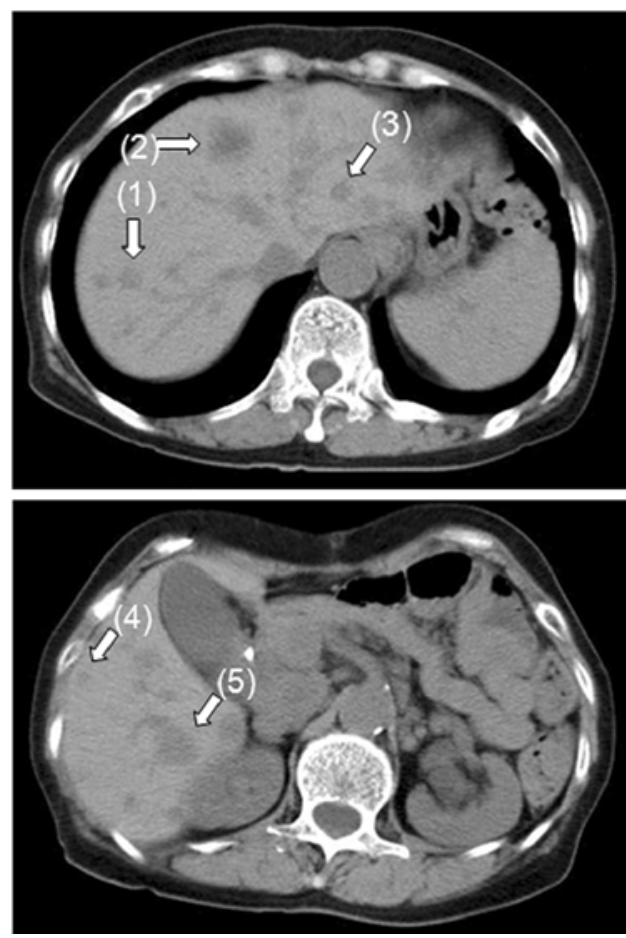

response was stable disease after two treatment courses, and remarkable reactions in the ELISPOT assay were noted, despite DTH reactions in all patients. Median survival of patients who had $\geq 100$ and $\leq 99$ specific spots in the ELISPOT assay was $296.6 \pm 41$ days and $117.5 \pm 40.0$ days, respectively. Thus, the presence of more specific spots was correlated with increased survival (Table 2). 
Fig. 4 Changes in evaluation of metastasis and carcinoembryonic antigen (CEA) in patient 1. Although CEA and the sum of the largest cross-sectional diameter of the evaluated liver metastases decreased initially, they increased thereafter

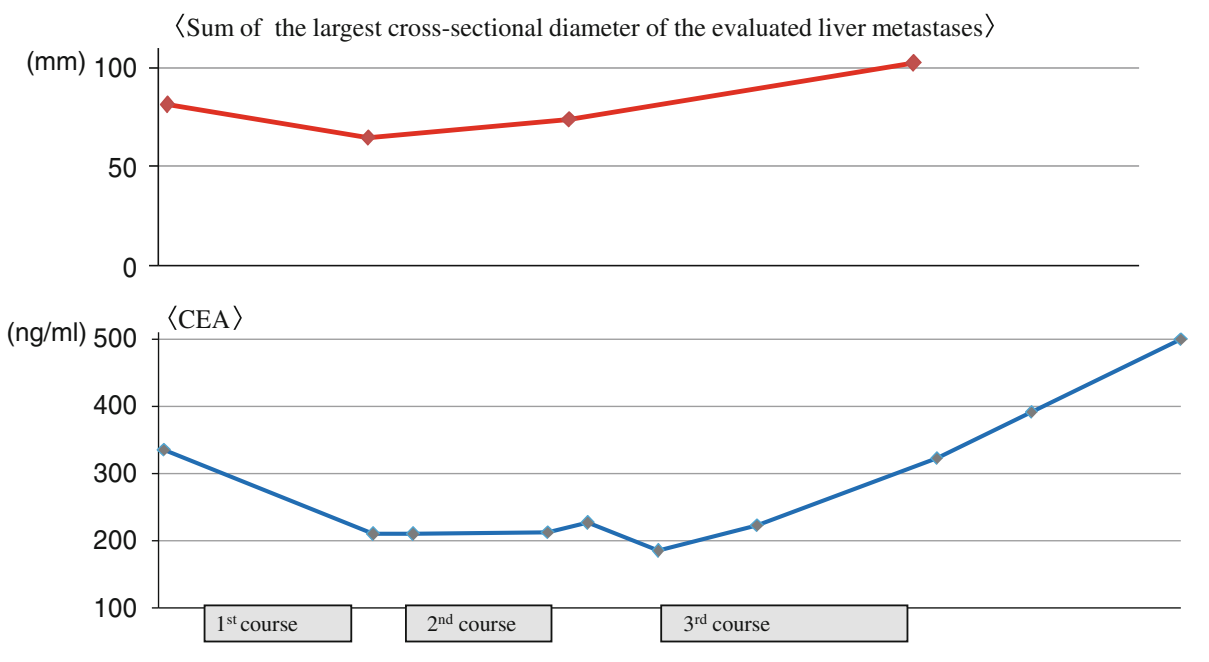

ELISPOT assay of patient 1

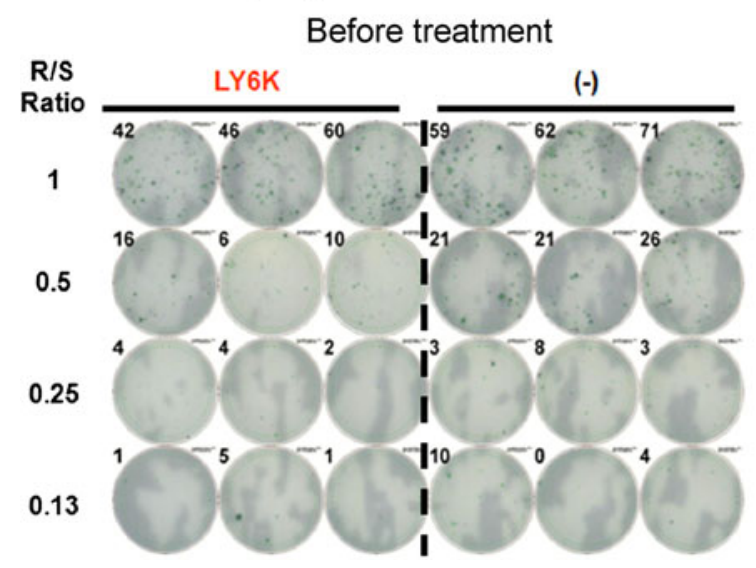

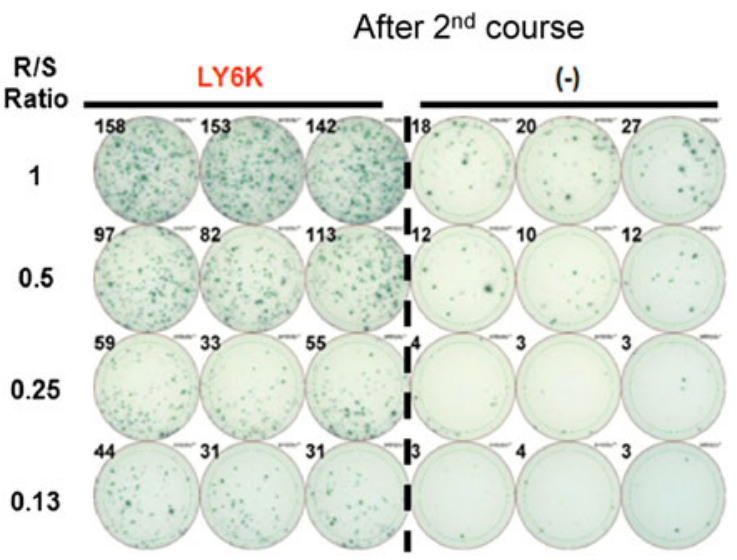

Fig. 5 In patient 1, the number of specific spots correlated with the R/S ratio and increased after vaccination

In patient 1, a significant antitumor effect was observed after one treatment course (Fig. 3). Figure 3 shows that evaluated tumors 1-4 decreased in size while tumor 5 became larger after the first treatment course. The sum of the largest cross-sectional diameter of the evaluated liver metastases decreased to $80.2 \%$ after one course compared with the pretreatment size and then increased to $91.3 \%$ the pretreatment size after two courses (Fig. 4). Unfortunately, the sum total of the tumor diameter continued to increase thereafter. The vaccine effect was therefore deemed not significant. However, the carcinoembryonic antigen (CEA) value at pretreatment and after the first, second courses was 335,209 , and $184 \mathrm{ng} / \mathrm{ml}$, respectively (Fig. 4). The CTL response in this patient after two courses is shown in Fig. 5. A significantly higher number of spots was observed after stimulation with LY6K-177 peptide-pulsed TISI cells than HIV-Env peptide-pulsed TISI cells.

\section{Discussion}

LY6K overexpression has been confirmed in several malignancies including head and neck squamous cell carcinomas as well as breast, lung, bladder, and esophageal cancers $[12,13,15-19]$. LY6K overexpression is associated with a poorer prognosis in patients with non-small cell lung carcinomas as well as those with esophageal squamous cell carcinomas [12]. Although the detailed function of LY6K is not known, its expression in normal tissue has been confirmed only in the testis [12]. This finding identifies LY6K as a promising target for immunotherapy. A clinical trial of vaccine therapy with LY6K as the target was previously reported [13, 14]; however, expression in gastric cancer has not yet been reported. We expected vaccine therapy with LY6K as the target tobe effective because immunohistochemistry confirmed expression in $85 \%$ of gastric cancers. 
In this protocol, the only adverse events were redness and induration of the skin. It was therefore concluded that therapy with LY6K-177 peptide/Montanide ISA 51 was well tolerated, as reported for other peptide vaccines with Montanide ISA 51 that revealed no more than three grades of adverse events [20-22].

The MOS in this study was 7.9 months. The survival time for gastric cancer patients receiving the best supportive care is $3-5$ months. In addition, although tumor reduction was observed in only one patient, these results are not inferior compared with those of reports of secondline chemotherapy for advanced gastric cancer [23-25]. The CTL response was significantly stronger in four patients, with the resulting prognosis also being longer than in the remaining two patients. Considering this, we can assume that the cellular immunity resulting from the vaccine demonstrated an antitumor effect. It is therefore believed that this clinical trial should advance to phase 2 .

Patient 1 received the peptide vaccine therapy without any concomitant treatment during the clinical trial. Vaccine treatment subsequently resulted in tumor shrinkage and reduced CEA values. However, despite these findings, some of the tumors continued to grow, and moreover, tumors that were initially reduced subsequently regrew. The reason for this continued growth may be that the cancer was heterogeneous and thus not all tumor cells presented the LY6Kderived peptide. In immunostaining, no tumors consisted of cells stained only by the antibody against LY6K. Therefore, use of multiple peptides in this peptide vaccine therapy may be advantageous. Such research was recently conducted [14]; however, a future multipeptide vaccine requires further research into the oncoantigens of gastric cancer. With regard to tumor regrowth, the dosing interval in this study was 1 week during the first and second treatment courses and 2 weeks for the third. In patient 1, the tumors showed regrowth during the third course, perhaps because of extension of the dosing interval. The frequency of peptide vaccine doses should therefore be examined, even though it has not been an issue to date.

In conclusion, we identified LY6K in $85 \%$ of gastric cancer tissues. Subsequent treatment with the HLA-A2402restricted LY6K-derived peptide, LY6K-177 peptide (RYCNLEGPPI)/Montanide ISA 51, in patients with progressing advanced gastric cancer was well tolerated and safe. Induction of an antigen-specific cellular immune response was observed in some patients, with a clear vaccine effect on tumor reduction in one patient. The findings suggest that this research should be advanced to phase 2 for the development of a treatment option for advanced gastric cancer.

Acknowledgments The authors thank Prof. Yusuke Nakamura, Dr. Takuya Tsunoda, Dr. Koji Yoshida, Mr. Ryuji Osawa, and Ms.
Sachiko Yoshimura, Laboratory of Molecular Medicine, Human Genome Center, Institute of Medical Science, the University of Tokyo, for their advice and cooperation and for providing the peptides.

Conflict of interest The authors declare no conflict of interest.

\section{References}

1. Parkin DM, Bray F, Ferlay J, Pisani P. Global cancer statistics, 2002. CA Cancer J Clin. 2005;55:74-108.

2. Parkin DM, Pisani P, Ferlay J. Estimates of the worldwide incidence of eighteen major cancers in 1985. Int J Cancer. 1993;54:594-606

3. Maruyama K, Kaminishi M, Hayashi K, Isobe Y, Honda I, Katai H, et al. Gastric cancer treated in 1991 in Japan: data analysis of nationwide registry. Japanese Gastric Cancer Association Registration Committee. Gastric Cancer. 2006;9:51-66.

4. Koizumi W, Kurihara M, Nakano S, Hasegawa K. Phase II study of S-1, a novel oral derivative of 5-fluorouracil, in advanced gastric cancer. For the S-1 Cooperative Gastric Cancer Study Group. Oncology. 2000;58:191-7.

5. Takiuchi H, Narahara H, Tsujinaka T, Gotoh M, Kawabe S, Katsu K, et al. Phase I study of S-1 combined with irinotecan (CPT-11) in patients with advanced gastric cancer (OGSG 0002). Jpn J Clin Oncol. 2005;35:520-525.

6. Yoshida K, Ninomiya M, Takakura N, Hirabayashi N, Takiyama $\mathrm{W}$, Sato Y, et al. Phase II study of docetaxel and S-1 combination therapy for advanced or recurrent gastric cancer. Clin Cancer Res. 2006;12:3402-7.

7. Kikuchi T, Daigo Y, Katagiri T, Tsunoda T, Okada K, Kakiuchi $\mathrm{S}$, et al. Expression profiles of non-small cell lung cancers on cDNA microarrays: identification of genes for prediction of lymph-node metastasis and sensitivity to anti-cancer drugs. Oncogene. 2003;22:2192-205.

8. Kakiuchi S, Daigo Y, Ishikawa N, Furukawa C, Tsunoda T, Yano $\mathrm{S}$, et al. Prediction of sensitivity of advanced non-small cell lung cancers to gefitinib (Iressa, ZD1839). Hum Mol Genet. 2004; $13: 3029$

9. Kikuchi T, Daigo Y, Ishikawa N, Katagiri T, Tsunoda T, Yoshida $\mathrm{S}$, et al. Expression profiles of metastatic brain tumor from lung adenocarcinomas on cDNA microarray. Int J Oncol. 2006;28:799.

10. Taniwaki M, Daigo Y, Ishikawa N, Takano A, Tsunoda T, Yasui $\mathrm{W}$, et al. Gene expression profiles of small-cell lung cancers: molecular signatures of lung cancer. Int J Oncol. 2006;29: 567-75.

11. Yamabuki T, Daigo Y, Kato T, Hayama S, Tsunoda T, Miyamoto $\mathrm{M}$, et al. Genome-wide gene expression profile analysis of esophageal squamous-cell carcinomas. Int J Oncol. 2006;28: $1375-84$.

12. Ishikawa $\mathrm{N}$, Takano $\mathrm{A}$, Yasui $\mathrm{W}$, Inai $\mathrm{K}$, Nishimura $\mathrm{H}$, Ito $\mathrm{H}$, et al. Cancer-testis antigen lymphocyte antigen 6 complex locus $\mathrm{K}$ is a serologic biomarker and a therapeutic target for lung and esophageal carcinomas. Cancer Res. 2007;67:11601-11.

13. Kono K, Mizukami Y, Daigo Y, Takano A, Masuda K, Yoshida $\mathrm{K}$, et al. Vaccination with multiple peptides derived from novel cancer-testis antigens can induce specific T-cell responses and clinical responses in advanced esophageal cancer. Cancer Sci. 2009;100:1502-9.

14. Iwahashi M, Katsuda M, Nakamori M, Nakamura M, Naka T, Ojima $\mathrm{T}$, et al. Vaccination with peptides derived from cancertestis antigens in combination with CpG-7909 elicits strong specific $\mathrm{CD} 8+\mathrm{T}$ cell response in patients with metastatic 
esophageal squamous cell carcinoma. Cancer Sci. 2010;101: 2510-7. doi:10.1111/j.1349-7006.2010.01732.x.

15. de Nooij-van Dalen AG, van Dongen GA, Smeets SJ, Nieuwenhuis EJ, Stigter-van Walsum M, Snow GB, et al. Characterization of the human Ly-6 antigens, the newly annotated member Ly-6K included, as molecular markers for head-andneck squamous cell carcinoma. Int J Cancer. 2003;103:768-74.

16. Scanlan MJ, Simpson AJ, Old LJ. The cancer/testis genes: review, standardization, and commentary. Cancer Immun. 2004;4:1.

17. Lee JW, Lee YS, Yoo KH, Lee KH, Park K, Ahn T, et al. LY-6K gene: a novel molecular marker for human breast cancer. Oncol Rep. 2006;16:1211-4.

18. Choi SH, Kong HK, Park SY, Park JH. Metastatic effect of LY6K gene in breast cancer cells. Int J Oncol. 2009;35:601-7.

19. Matsuda R, Enokida H, Chiyomaru T, Kikkawa N, Sugimoto T, Kawakami K, et al. LY6K is a novel molecular target in bladder cancer on basis of integrate genome-wide profiling. $\mathrm{Br} \mathrm{J}$ Cancer. 2011;104:376-86.

20. Kawakami Y, Fujita T, Matsuzaki Y, Sakurai T, Tsukamoto M, Toda $\mathrm{M}$, et al. Identification of human tumor antigens and its implications for diagnosis and treatment of cancer. Cancer Sci. 2004;95:10.

21. Yataro D, Yusuke N. From cancer genomics to thoracic oncology: discovery of new biomarkers and therapeutic targets for lung and esophageal carcinoma. Gen Thorac Cardiovasc Surg. 2008;56:43-53.

22. Slingluff CL Jr, Yamshchikov G, Neese P, Galavotti H, Eastham $\mathrm{S}$, Engelhard VH, et al. Phase I trial of a melanoma vaccine with gp100280-288 peptide and tetanus helper peptide in adjuvant: immunologic and clinical outcomes. Clin Cancer Res. 2001;7: 3012-24.

23. Scartozzi M, Galizia E, Verdecchia L, Berardi R, Antognoli S, Chiorrini S, et al. Chemotherapy for advanced gastric cancer: across the years for a standard of care. Expert Opin Pharmacother. 2007;8:797-808. doi:10.1517/14656566.8.6.797.

24. Wilson D, Hiller L, Geh JI. Review of second-line chemotherapy for advanced gastric adenocarcinoma. Clin Oncol (R Coll Radiol). 2005;17:81-90. doi:10.1016/j.clon.2004.10.006.

25. Wohrer SS, Raderer M, Hejna M. Palliative chemotherapy for advanced gastric cancer. Ann Oncol. 2004;15:1585-95. doi: 10.1093/annonc/mdh422. 